\title{
Reliability in Extreme Isolation: A Natural Language Processing Tool for Stress Self-assessment
}

\author{
Alessandro Alcibiade ${ }^{1(\bowtie)}$, Irene Lia Schlacht ${ }^{2}$, Francesco Finazzi ${ }^{3}$, \\ Massimiliano Di Capua ${ }^{5}$, Giacomo Ferrario ${ }^{6}$, Giorgio Musso ${ }^{4}$, \\ and Bernard Foing ${ }^{7}$ \\ 1 Università di Pisa, Pisa, Italy \\ alessandro.alcibiade@marsplanet.org \\ ${ }^{2}$ HMKW University and PoliMi, Milan, Italy \\ schlacht@mailbox.org \\ 3 E-Campus Milano, Milan, Italy \\ francescofinazzi5@gmail.com \\ 4 Thales Alenia Italy, Milan, Italy \\ Giorgio.Musso@thalesaleniaspace.com \\ ${ }^{5}$ University of Rome, Rome, Italy \\ maxdicapua@gmail.com \\ ${ }^{6}$ Università degli studi di Pavia, Pavia, Italy \\ giacomo. ferrario0l@gmail.com \\ ${ }^{7}$ ILEWG at ESTEC ESA, Noordwijk, The Netherlands \\ Bernard.Foing@esa.int
}

\begin{abstract}
Life in isolation is a condition that affects more people every day. From isolation of people in case of an epidemic (Ebola, Coronavirus, ..) to mineworkers or scientist in the Antarctic environment, it is currently well established that extreme isolation is a stressor element which negatively affects human creating stress and social conflict. Living in outer space is one of the most extreme forms of isolation that can be used as a test bench for isolation research. Crews in space are expected to be able to manage independently stress and social conflict problems to be highly reliable and autonomous. Human natural language (NL) has the potential of being a valuable source of information on crew-members stress and reliability in isolation. Based on the use of NL, the PSI (Performance and Stress in Isolation) research group is developing an Artificial Intelligent tool for the autonomous monitoring of stress and reliability. In order to develop this tool, the first step (here presented) is to investigate the correlation of NL with stress and reliability within the PSI experiment. The experiment consisted of three tasks to be performed periodically during the isolation: 1. Analysis of free written communication (NL); 2. Short selfreference questionnaire; and 3. Detection of heart rate and blood pressure values. In this paper we present the preliminary data collected from 29 participants (17 males and 12 females) to 6 space analogue missions accomplished in Europe, the USA and Israel in the 2016 - 2019 time frames. That is, so far, the largest database ever analyzed of this kind. The results are coherent with what is described in the literature about NLP content and style analysis, with the novelty of the correlations found with the cardiovascular parameters, thus reinforcing the
\end{abstract}


perspective of applying a NLP AI system for supporting stress management in extreme isolation contexts from Space to Earth spin off.

Keywords: Human factors $\cdot$ Human-systems integration $\cdot$ Cognitive neurosciences $\cdot$ Natural language processing $\cdot$ Artificial intelligence $\cdot$ Space psycho-physiology $\cdot$ Space spin off

\section{Introduction}

In every mission isolation is a stressor element which negatively affects human behaviour, mood and cognitive performances, creating stress and social conflict. Living in outer space is one of the most extreme form of isolation that can be used as test bench for spin off of isolation research on Earth. Future crewed long lasting space missions Beyond Low Earth Orbit (BEO) pose danger and challenges not previously faced by any human explorer. Not only would the trip's length far exceed any previous mission but the crew members would also experience significant communication delays, endure lengthy periods of inactivity punctuated by crises and they would lose sight of the Earth as they journey towards deep space. The effects of these unique circumstances on astronauts are still not completely described but they will likely test human psycho-physiological limits like never before and the importance of being able to self-monitoring the psychological and physiological factors will be crucial to their success [1].

In particular the relative lack of human factors data Behond Eath Orbit (BEO) creates a significant difficulty in understanding what the problems are and how to address them in the context of a crewed mission with associated constraints. What is already well described into the scientific literature is how "extreme" environmental stimuli give origin to an adaptive response, which comprehends systemic and behavioural changes aimed to develop the best homeostatic capability of the individual, thus increasing his survival chances. These adaptive changes are produced by chemical mediators, such as adrenalin, glucocorticoids, growth hormone and cytokines, that act on specific receptors localized in different organs. Chronic stress can produce the so called allostatic state, characterized by an increased activity of the mediators on their target cells that leads to receptor desensitization and tissue damages, thus connoting the allostatic load [2]. This may cause dramatic consequences, all detrimental in the operating scenario of a crewed space mission that include insomnia, depression, cognitive impairment and various cardiovascular diseases.

Because of all those assumptions it is now well established that crewed missions BEO are expected to be highly autonomous and automated in order to improve the human factor on the overall outcome of the mission and also, because of the communication delay with the earth station that imposes the spacecraft to be as self-reliant as possible in the accomplishment of its tasks. From these findings comes the need to design and implement more advanced systems of Artificial Intelligence (AI) and human-computer interfaces on board future spaceships. An effective and innovative human-computer interface cannot do without the implementation of the possibility of making astronauts communicate with $\mathrm{AI}$ in the most spontaneous way for humans: 
natural language. The branch of computer science that deals with "teaching" AI machines how to effectively communicate in the natural language of humans is called Natural Language Processing (NLP).

Other than just potentially being a very effective human-machine interface, human natural language has the potential of being a valuable source of information regarding crew-members psycho-physiological well-being, stress and reliability. In fact, it has been well known for more than a century that psychological states and processes are encoded in verbal behavior and psychological constructs may be reliably explored by text analysis [3, 4]. A multitude of studies indicate the existence of a strong correlation between the variation of the word spectrum used in an individual's daily vocabulary in a given period and the variation in his level of physiological and psychological stress [5-7]. The aim of this study was to investigate the prospect of using NLP to monitor the psychological wellbeing and stressful conditions of personnel undergoing prolonged missions in Isolated and Confined Environments (ICEs). NLP is a theorymotivated range of computational techniques for the automatic analysis and representation of human language [8]. The history of NLP began in the 1950s as the intersection of artificial intelligence and linguistics [8]. In recent years, increases in computing power have led to the development of sophisticated machine learning algorithms that allow researchers to analyze enormous quantities of biomedical data to detect covered patterns [9]. These developments have brought back the growing of the studies of human Natural Language from a psychometric perspective, consequently directing the progression of NLP algorithms as a diagnostic tool for psychiatric and psychological disorders based on the analysis of written and spoken language [10].

The studies of potential space applications of Natural Language Processing (NLP) have been pioneered by the Russian IBMP research group led by Prof. Vadim Gushim, who analyzed texts extracted from Space Stations (MIR and ISS) and Analogue Missions (MARS500 and MARS105) crews communications with the respective ground controls. A computerized content analysis of written daily reports of the MARS105 experiment crew (IBMP, Moscow, 2009) revealed different tendencies in communicating behaviour. A stress-related strategy was "closing the communication channel" [11], which was found to correlate with elevated urinary cortisol level. These results suggest as both language use and somatic markers can have a diagnostic value in identifying stressful conditions and changes thereof.

Current NLP analyses typically consist of both a content analysis assessing what a subject says and a style analysis evaluating how subjects express themselves. So far, the strongest associations according the scientific literature regarding content analysis have identified in the first-person singular personal pronouns the strongest NLP content indicator of depressive mood [12]. Adjectives and adverbs expressing negative emotions are found to be strongly linked to depressive mood and suicidal thoughts $[13,14]$. Regarding style analysis, it has been found that individuals with suicidal thoughts, anxiety or depressive mood tend to use more absolutist words than the general population, thus expressing ideas of certainty [12].

This study explores the possibility to use the NLP to identify language markers that could be of diagnostic value in assessing crew-members psychological wellbeing and stress. Moreover for the first time in literature, it looks for new correlations among: NLP-markers and known stress related cardio-vascular parameters such as Heart Rate, 
and NLP-markers and a self-reference psychometric short questionnaire. Those findings could prospectively lead to the development of an AI learning tool to monitor remotely and non-invasively not only the astronauts' wellbeing and cognitive performance status in future missions BEO but also on different spin off isolation context on Earth.

\section{Materials and Methods}

The PSI (Performance \& Stress in Isolation) experiment aims to analyze stress levels during extreme missions without intrusive instruments. It has received the approval of the Ethics Committee of the Pope Giovanni XXIII Hospital in Bergamo on 11 August 2017. In this paper, the preliminary data collected from 29 participants (17 males and 12 females) to 6 space analogue missions (Figs. 1 and 2) accomplished among Europe, the USA and Israel in the 2016-2019 time frames, are presented. That is, as yet, the largest database ever analyzed of this kind.

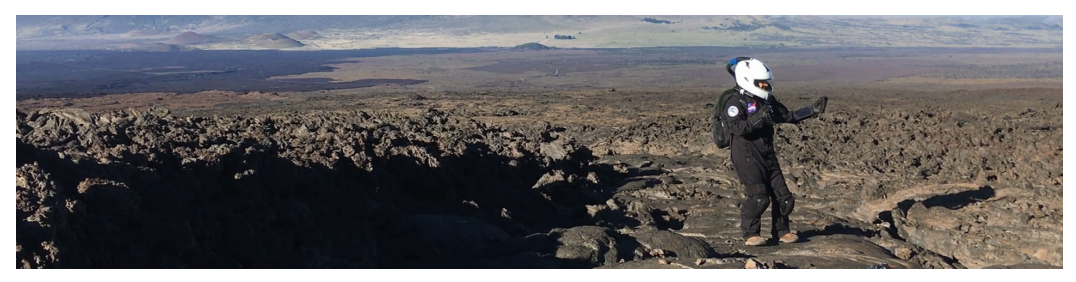

Fig. 1. Astronaut simulation, Female crew mission. (c) Dr. M. Musilova, EMMIHS 2019 (ILEWG EuroMoonMars-International Moonbase Alliance- HISEAS)

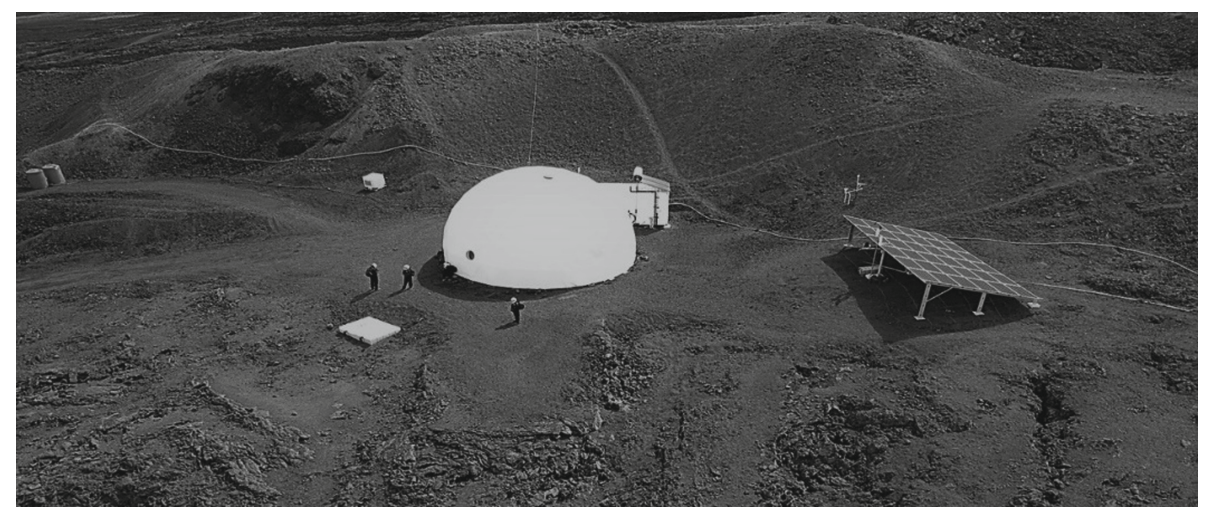

Fig. 2. HI SEAS space simulation base in Hawaii. (c) EMMIHS 2020 (ILEWG EuroMoonMars-International Moonbase Alliance- HISEAS) 
The core of the PSI experiment consisted of three tasks to be performed periodically during the isolation:

1. Short text collection, to be analyzed through a NLP software. Every crew member was asked to write in a maximum of 30 min a text with a maximum of 500 words in his mother tongue (e.g. diary collection or daily and official mission report if not affected by a strong imposed structure).

a. Short self reference questionnaire, to assess subjects' perceived psychological stress levels. It consisted of the following three questions:

b. Q1: "How stressed do you feel today?" (from low:1 to high:10);

c. Q2: "How well were you able to perform today?" (from low:1 to high:10);

d. Q3: "How comfortable do you feel today?" (from low:1 to high:10).

2. Detection of Heart Rate (HR) and Blood Pressure (systolic, diastolic and mean arterial pressure) values, as markers of subjects physiological stress levels. The measurements were taken using an OMRON M3 device, provided to every crew, and crew members were instructed to measure the parameters according to the latest American Heart Association recommendations that at least two readings be taken, with a one-minute interval between them and the average of the measurements recorded.

For each subject the written texts were categorized based on the editing date and time spent in isolation and according to the subject's gender and mother tongue as well. This research relied on the use of two content analysis software tools. One is an Italian tool specifically tailored to this scope. The second tool is LIWC (Linguistic Inquiry and Word Count), developed by the Pennebaker Lab [15].

The data was analyzed using standardized z-score output of linguistic markers from LIWC software as well as using our own specifically tailored software which provides two variables not considered by the LIWC software: the number of Different Words in a text (DW, assumed to be an index of verbal fluency) and the number of Average Characters per Word in a text $(\mathrm{CW}$, assumed to be an index of verbal and cognitive complexity). A Pearson's correlation analysis was run to assess the strength of relationships between linguistic markers.

\section{Results}

Statistical analysis correlations were searched between the NLP parameters and the answers to the self-reference psychological questionnaire and the cardiovascular parameters. Numerous novel correlations were identified; those lead an interest for a confirmation with a larger number of subjects. Here will be illustrated the most relevant correlations (Fig. 3). 

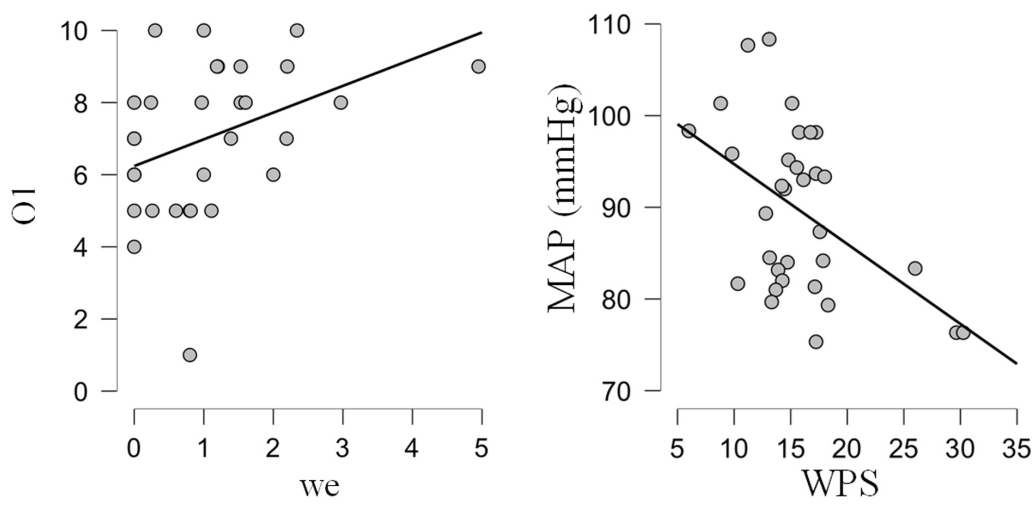

Fig. 3. Left: Scatter Plot of the First Person Plural Pronouns usage (we) and the average scoring at the first question of the self reference test (Q1) $(\mathrm{p}=.002, \mathrm{r}=.490)$. Right: (right) Scatter Plot of the average number of Words per Sentence (WPS) and the Mean Arterial Pressure (MAP) values $(\mathrm{p}=.013, \mathrm{r}=-.393)$.

\subsection{NLP Parameters and Perceived Stress}

A strong direct correlation emerged at the Person Regression analysis $(\mathrm{R}=0.490$, $\mathrm{p}=0.002$ ) between the frequency of first person plural pronouns in a text and the scoring at the first questions of the self-reference psychological test ("How stressed do you feel today?"). Perceived stress is thus directly correlated to the subject's tendency to express using the first person plural pronouns, which is linked to a feeling of detachment, selflessness and isolation [15].

\subsection{NLP and Cardiovascular Parameters}

A moderate negative correlation was found between the average number of Words per Sentence (WPS) and the Mean Arterial Pressure (MAP) values $(\mathrm{R}=-0.393$, $\mathrm{p}=0.013$ ). WPS is considered as an indicator of subjects' cognitive complexity [15], while the MAP is an estimation of the average blood pressure in an individual during a single cardiac cycle. It is widely described in the scientific literature the link between higher blood pressure values and chronic $[16,17]$, allowing us to assume that in individuals in extreme isolation higher MAP values are an physiological indicator of stress and that are negatively correlated to subjects' cognitive performances.

\section{Discussion and Conclusion}

The results confirm the literatures about NLP content and style analysis, with the important novelty of the correlation hypotheses between the NLP and cardiovascular parameters. Being well assessed in the scientific literature $[15,16]$ how higher blood pressure values are linked to chronic stress and its complications, this finding open up the perspective of developing a NLP AI system to self-monitor not only astronauts' 
psychological and cognitive well being but also of their overall psycho-physiological status with spin off applications in different isolation contexts on Earth.

Furthermore we know that the literature review is coherent in assessing that human NLP-psycho-physiological parameters correlations do not always follow the same trends within every subject, like on most parts of the human psycho-physiological phenomena [10-15]. The reason is that NLP and stress-related parameters are highly influenced by many factors such as subjects' age, cultural background, education-training, gender and length of the isolation. The implementation of NLP in a software designed to let crew members to monitor their wellbeing in isolation, cannot be based on a single rigid algorithm but should be based on a machine learning tool trained to identify the peculiar pattern followed by each parameter in a given subject.

Finally, due to the restricted number of subjects enrolled in this study, the statistical power of this analysis is low and further research is needed to confirm specific linguistic indicators of functionally prohibitive stress responses.

Acknowledgments. All the people and entities involved such as: Arch. G. Musso for setting up the session on space at AHFE, all the people involved in the space analogues simulations for their commitment in trying to push forward the frontiers of human knowledge, all the people that support the data collection on the next Antarctic missions, ILEWG, A. Kołodziejczyk of Poland Analog Astronaut Training Center, R. Sorek Abramovich of the Dead Sea and Arava Science Center in Israel, Prof. P. del Leon from University of North Dakota UND space study and the people involved on the ILEWG EuroMoonMars, Lunares, ILMAH (UND), PMARS, MDRS, DMARS missions.

\section{References}

1. Schlacht, I.L.: Space Habitability. Tu-Berlin, Germany (2012). www.extreme-design.eu/doc/ 2012-PhD-Schlacht.pdf

2. McEwen, B.S.: Sleep deprivation as a neurobiologic and physiologic stressor: allostasis and allostatic load. Metabolism 55, S20-S23 (2006)

3. Osgood, C.E., Walker, G.: Motivation and language behavior: a content analysis of suicide notes. J. Abnorm. Psychol. 59, 58-67 (1959)

4. Freud, S.: The Psychopathology of Everyday Life. W.W. Norton \& Co., New York (1901)

5. Allegrini, P., Grignolini, P., Palatella, L.: Intermittency and scale-free networks: a dynamical model for human language complexity. Chaos Solitons Fractals. 20, 95-105 (2003)

6. Ferrer i Cancho, R.: The variation of Zipf's law in human language. Eur. Phys. J. B 44, 249257 (2005)

7. Piantadosi, S.T.: Zipf's word frequency law in natural language: a critical review and future directions. Psychon. Bull. Rev. 21(5), 1112-1130 (2014)

8. Cambria, E., White, B.: Jumping NLP curves: a review of natural language processing research. IEEE Comput. Intell. Mag. 9, 48-57 (2014)

9. Nadkarni, P.M., Ohno-Machado, L., Chapman, W.W.: Natural language processing: an introduction. J. Am. Med. Inform. Assoc. 18(5), 544-551 (2011)

10. Tausczik, Y.R., Pennebaker, J.W.: The psychological meaning of words: LIWC and computerized text analysis methods. J. Lang. Soc. Psychol. 29, 24-54 (2010) 
11. Yusupova, A., Ushakov, I., Gushin, V.: Communications in long-term space flights and space simulations. Content analysis as a source of information about personality during space flight. LAMBERT Academic Publishing, Germany (2011)

12. Al-Mosaiwi, M., Johnstone, T.: Linguistic markers of moderate and absolute natural language. Pers. Individ. Differ. 134, 119-124 (2018)

13. Fischer, U., McDonnell, L., Orasanu, J.: Linguistic correlates of team performance: toward a tool for monitoring team functioning during space missions. Aviat. Space Environ. Med. 78, B86-B95 (2007)

14. Ramirez-Esparza, N.: The psychology of word use in depression forums in English and in Spanish: testing two text analytic approaches. Association for the Advancement of Artificial Intelligence (2015). www.cs.columbia.edu/ julia/papers/Ramirez-Esparzaetal08.pdf

15. Pennebaker, J.W., et al.: The development and psychometric properties of LIWC2015. University of Texas at Austin, Austin, TX (2015). http://liwc.wpengine.com/

16. Ushakov, A.V., Ivanchenko, V.S., Gagarina, A.A.: Psychological stress in pathogenesis of essential hypertension. Curr. Hypertens. Rev. 12(3), 203-214 (2016)

17. Kennelly, S.P., Lawlor, B.A., Kenny, R.A.: Blood pressure and dementia: a comprehensive review. Ther. Adv. Neurol. Disord. 2(4), 241-260 (2009) 